Vladikavkaz Mathematical Journal 2021, Volume 23, Issue 3, P. 80-90

УДК 517.98

DOI 10.46698/w5172-0182-0041-c

\title{
PARTIAL INTEGRAL OPERATORS OF FREDHOLM TYPE ON KAPLANSKY-HILBERT MODULE OVER $L_{0}$
}

\author{
Yu. Kh. Eshkabilov ${ }^{1}$ and R. R. Kucharov ${ }^{2}$ \\ ${ }^{1}$ Karshi State University, 17 Kuchabag St., Karshi 180117, Uzbekistan; \\ ${ }^{2}$ National University of Uzbekistan, 4 University St., Tashkent 100174, Uzbekistan \\ E-mail: ramz3364647@yahoo.com, yusup62@mail.ru
}

\author{
Dedicated to the 80th anniversary \\ of Professor Stefan Grigorievich Samko
}

\begin{abstract}
The article studies some characteristic properties of self-adjoint partially integral operators of Fredholm type in the Kaplansky-Hilbert module $L_{0}\left[L_{2}\left(\Omega_{1}\right)\right]$ over $L_{0}\left(\Omega_{2}\right)$. Some mathematical tools from the theory of Kaplansky-Hilbert module are used. In the Kaplansky-Hilbert module $L_{0}\left[L_{2}\left(\Omega_{1}\right)\right]$ over $L_{0}\left(\Omega_{2}\right)$ we consider the partially integral operator of Fredholm type $T_{1}\left(\Omega_{1}\right.$ and $\Omega_{2}$ are closed bounded sets in $\mathbb{R}^{\nu_{1}}$ and $\mathbb{R}^{\nu_{2}}, \nu_{1}, \nu_{2} \in \mathbb{N}$, respectively). The existence of $L_{0}\left(\Omega_{2}\right)$ nonzero eigenvalues for any selfadjoint partially integral operator $T_{1}$ is proved; moreover, it is shown that $T_{1}$ has finite and countable number of real $L_{0}\left(\Omega_{2}\right)$-eigenvalues. In the latter case, the sequence $L_{0}\left(\Omega_{2}\right)$-eigenvalues is order convergent to the zero function. It is also established that the operator $T_{1}$ admits an expansion into a series of $\nabla_{1}$-one-dimensional operators.
\end{abstract}

Key words: partial integral operator, Kaplansky-Hilbert module, $L_{0}$-eigenvalue.

Mathematical Subject Classification (2010): 45A05, 47A10, 47G10, 45P05, 45B05, 45C05.

For citation: Eshkabilov, Yu. Kh. and Kucharov, R. R. Partial Integral Operators of Fredholm Type on Kaplansky-Hilbert Module over $L_{0}$, Vladikavkaz Math. J., 2021, vol. 23, no. 3, pp. 80-90. DOI: 10.46698/w5172-0182-0041-c.

\section{Introduction}

Linear equations and operators involving partial integrals appear in elasticity theory, continuum mechanics, aerodynamics and in PDE theory [1]. Self-adjoint partial integral operators arise in the theory of Schrodinger operators [2,3]. Spectral properties of a discrete Schrodinger operator $H$ are closely related (see $[3,4]$ ) to the partial integral operators which participate in the presentation of operator $H$.

Let $\Omega_{1}$ and $\Omega_{2}$ be closed bounded subsets in $\mathbb{R}^{\nu_{1}}$ and $\mathbb{R}^{\nu_{2}}$, respectively. Partial integral operator (PIO) of Fredholm type in the space $L_{p}\left(\Omega_{1} \times \Omega_{2}\right), p \geqslant 1$, is an operator of the form [1]

$$
T=T_{0}+T_{1}+T_{2}+K,
$$

where operators $T_{0}, T_{1}, T_{2}$ and $K$ are defined by the following formulas

$$
T_{0} f(x, y)=k_{0}(x, y) f(x, y),
$$

(C) 2021 Eshkabilov, Yu. Kh. and Kucharov, R. R. 


$$
\begin{gathered}
T_{1} f(x, y)=\int_{\Omega_{1}} k_{1}(x, s, y) f(s, y) d s, \\
T_{2} f(x, y)=\int_{\Omega_{2}} k_{2}(x, t, y) f(x, t) d t, \\
K f(x, y)=\iint_{\Omega_{1}} \int_{\Omega_{2}} k(x, y ; s, t) f(s, t) d s d t .
\end{gathered}
$$

Here $k_{0}, k_{1}, k_{2}$ and $k$ are given measurable functions on $\Omega_{1} \times \Omega_{2}, \Omega_{1}^{2} \times \Omega_{2}, \Omega_{1} \times \Omega_{2}^{2}$ and $\left(\Omega_{1} \times \Omega_{2}\right)^{2}$, respectively, and all integrals have to be understood in the Lebesgue sense, where $d s=d \mu_{1}(s), d t=d \mu_{2}(t), \mu_{k}(\cdot)$ - the Lebesgue measure on the $\sigma$-algebra of subsets $\Omega_{k}$, $k=1,2$.

Furthermore, some simple solvability conditions for the equations $T f=g$ were investigated by several authors (see, for example, [1] and its references). Spectral properties of the given operator has been studied in $[1,4,5]$.

Nevertheless, the description of the spectra of self-adjoint PIOs with $L_{2}$ kernels remains an open question. Difficulty of this problem is connected with non-compactness of the operators $T_{1}$ and $T_{2}$. The article studies some characteristic properties of self-adjoint partially integral operators of Fredholm type in the Kaplansky-Hilbert module $L_{0}\left[L_{2}\left(\Omega_{1}\right)\right]$ over $L_{0}\left(\Omega_{2}\right)$. The mathematical tools from the Kaplansky-Hilbert module is used as presented in [6].

The paper is organized as follows. In Section 3 we prove the existence of an $L_{0}$-eigenvalue for the PIO $T_{1}$.

In Section 4 we study existence of the countable consequence of real $L_{0}$-eigenvalues for PIO $T_{1}$. In Section 5 it is given the decomposition of the PIO $T_{1}$ in series of $\nabla_{1}$-one-dimensional operators. In Section 5 (in section 6) is given decomposition of the PIO $T_{1}$ (the PIO $T_{2}$ ) in series of $\nabla_{1^{-}}\left(\nabla_{2^{-}}\right)$one-dimensional operators.

\section{Kaplansky-Hilbert Module over $L_{0}$}

Recall some notions and results from the theory of Kaplansky-Hilbert modules (see [6]).

Let $\left(\Omega_{k}, \Sigma_{k}, \mu_{k}\right)$ be a space with complete finite measure $\mu_{k}, L_{0}\left(\Omega_{k}\right)$-algebra of equivalence classes of all complex measurable functions on $\left(\Omega_{k}, \Sigma_{k}, \mu_{k}\right)$, where $k=1,2$. We denote by $L_{0}\left[L_{2}\left(\Omega_{1}\right)\right]$ the set of equivalence classes of all complex measurable functions $f(x, y)$ on $\Omega_{1} \times$ $\Omega_{2}$, which satisfies the condition: the integral

$$
\varphi(y)=\int_{\Omega_{1}}|f(x, y)|^{2} d \mu_{1}(x)
$$

exists for almost all $y \in \Omega_{2}$ and $\varphi \in L_{0}\left(\Omega_{2}\right)$.

We consider the map $\langle\cdot, \cdot\rangle_{1}: L_{0}\left[L_{2}\left(\Omega_{1}\right)\right] \times L_{0}\left[L_{2}\left(\Omega_{1}\right)\right] \rightarrow L_{0}\left(\Omega_{2}\right)$ by rule

$$
\langle f, g\rangle_{1}=\int_{\Omega_{1}} f(s, y) \overline{g(s, y)} d \mu_{1}(s) .
$$

It is clear, that the map $\langle\cdot, \cdot\rangle_{1}$ satisfies the conditions of $L_{0}\left(\Omega_{2}\right)$-valued inner product.

For each $f \in L_{0}\left[L_{2}\left(\Omega_{1}\right)\right]$ we define $L_{0}$-norm:

$$
\|f\|_{1}(\omega)=\sqrt{\langle f, f\rangle_{1}(\omega)} .
$$

Then $L_{0}\left[L_{2}\left(\Omega_{1}\right)\right]$ is Banach-Kantorovich space over $L_{0}\left(\Omega_{2}\right)[6,7]$. Consequently, the space $L_{0}\left[L_{2}\left(\Omega_{1}\right)\right]$ is Kaplansky-Hilbert module over $L_{0}\left(\Omega_{2}\right)$ with the inner product $\langle\cdot, \cdot\rangle_{1}(\omega)$. 
If for the map $A: L_{0}\left[L_{2}\left(\Omega_{1}\right)\right] \rightarrow L_{0}\left[L_{2}\left(\Omega_{1}\right)\right]$ the equality $A(\alpha \cdot f+\beta \cdot g)=\alpha \cdot A f+\beta \cdot A g$ is hold for all $\alpha, \beta \in L_{0}\left(\Omega_{2}\right), f, g \in L_{0}\left[L_{2}\left(\Omega_{1}\right)\right]$, then $A$ is called $L_{0}\left(\Omega_{2}\right)$-linear operator.

If for the $L_{0}\left(\Omega_{2}\right)$-linear operator $A$ there exists $C=C(\omega) \in L_{0}\left(\Omega_{2}\right)$ such that, $\|A f\|_{1}(\omega) \leqslant$ $C(\omega)\|f\|_{1}(\omega)$ for all $f \in L_{0}\left[L_{2}\left(\Omega_{1}\right)\right]$, then $A$ is called $L_{0}\left(\Omega_{2}\right)$-bounded operator.

For each $L_{0}\left(\Omega_{2}\right)$-linear $L_{0}\left(\Omega_{2}\right)$-bounded operator $A$ we define $L_{0}\left(\Omega_{2}\right)$-norm by the rule

$$
\|A\|_{1}=\|A\|_{1}(\omega)=\sup \left\{\|A f\|_{1}(\omega):\|f\|_{1} \leqslant \mathbf{e}\right\} .
$$

We say the net $\left(\xi_{\alpha}\right)_{\alpha \in A} \subset L_{0}\left(\Omega_{2}\right)$ (o)-converges to the element $\xi \in L_{0}\left(\Omega_{2}\right)$, whenever there is a decreasing net $\left(e_{\beta}\right)_{\beta \in B} \subset L_{0}\left(\Omega_{2}\right)$ such that $\inf \left\{e_{\beta}: \beta \in B\right\}=\theta$ and for each $\beta \in B$ there is an index $\alpha(\beta) \in A$ with $\left|\xi_{\alpha}-\xi\right| \leqslant e_{\beta}$ for all $\alpha \in A: \alpha(\beta) \leqslant \alpha$. In this case, the element $\xi$ is called $(o)$-limit of the set $\left(\xi_{\alpha}\right)_{\alpha \in A}$ and we write $\xi=(o)-\lim \xi_{\alpha}$.

We know [8], that the (o)-converges of the net $\left(\xi_{\alpha}\right)_{\alpha \in A} \subset L_{0}\left(\Omega_{2}\right)$ to the element $\xi$ is equivalent to converges almost everywhere to the element $\xi$ of the net $\left(\xi_{\alpha}\right)_{\alpha \in A} \subset L_{0}\left(\Omega_{2}\right)$.

The net $\left(f_{\alpha}\right)_{\alpha \in A}$ in $L_{0}\left[L_{2}\left(\Omega_{1}\right)\right]$ is called (bo)-converging to $f \in L_{0}\left[L_{2}\left(\Omega_{1}\right)\right]$, if $(o)-\lim \left\|f_{\alpha}-f\right\|_{1}=\theta$ in $L_{0}\left(\Omega_{2}\right)$.

Let $\Lambda_{2}$ be the Boolean algebra of idempotents in $L_{0}\left(\Omega_{2}\right)$. If $\left(f_{\alpha}\right)_{\alpha \in A} \subset L_{0}\left[L_{2}\left(\Omega_{1}\right)\right]$ and $\left(\pi_{\alpha}\right)_{\alpha \in A}$ is a partition of the unit in $\Lambda_{2}$, then the series $\sum_{\alpha} \pi_{\alpha} \cdot f_{\alpha}$ (bo)-converges in $L_{0}\left[L_{2}\left(\Omega_{1}\right)\right]$ and its sum is called the mixing of $\left(f_{\alpha}\right)_{\alpha \in A}$ with respect to $\left(\pi_{\alpha}\right)_{\alpha \in A}$. We denote this sum by $\operatorname{mix}\left(\pi_{\alpha} f_{\alpha}\right)$. A subset $K \subset L_{0}\left[L_{2}\left(\Omega_{1}\right)\right]$ is called cyclic, if $\operatorname{mix}\left(\pi_{\alpha} f_{\alpha}\right) \in K$ for each $\left(f_{\alpha}\right)_{\alpha \in A} \subset K$ and any partition of the unit $\left(\pi_{\alpha}\right)_{\alpha \in A}$ in $\Lambda_{2}$. A subset $K \subset L_{0}\left[L_{2}\left(\Omega_{1}\right)\right]$ is called cyclically compact, if $K$ is cyclic and every net in $K$ has a cyclic subset that (bo)-converges to some point of $K$. A subset is called relatively cyclically compact, if it is contained in a cyclically compact set.

A $L_{0}$-linear operator in $L_{0}\left[L_{2}\left(\Omega_{1}\right)\right]$ is called cyclically compact, if for every $L_{0}$-bounded set $B$ in $L_{0}\left[L_{2}\left(\Omega_{1}\right)\right]$ the set $A(B)$ is relatively cyclically compact in $L_{0}\left[L_{2}\left(\Omega_{1}\right)\right]$.

Let $T_{1}$ be an operator in the Kaplansky-Hilbert module $L_{0}\left[L_{2}\left(\Omega_{1}\right)\right]$ over $L_{0}\left(\Omega_{2}\right)$ given by the formula

$$
\left(T_{1} f\right)(x, y)=\int_{\Omega_{1}} k_{1}(x, s, y) f(s, y) d \mu_{1}(s) .
$$

Here, $k_{1}(x, s, y)$ is a measurable function on $\Omega_{1}^{2} \times \Omega_{2}$.

Let the kernel $k_{1}(x, s, y)$ of the integral operator $T_{1}$ satisfy the condition

$$
\int_{\Omega_{1}} \int_{\Omega_{1}}\left|k_{1}(x, s, y)\right|^{2} d \mu_{1}(s) d \mu_{1}(x) \in L_{0}\left(\Omega_{2}\right) .
$$

Then, the operator $T_{1}$ with values in $L_{0}\left(\Omega_{2}\right)$ is linear and bounded on $L_{0}\left[L_{2}\left(\Omega_{1}\right)\right]$.

Also, let the kernel $k_{1}(x, s, y)$ satisfy the condition:

$$
k_{1}(x, s, y)=\overline{k_{1}(s, x, y)} .
$$

Then the operator $T_{1}$ is a self-adjoint operator on the Kaplansky-Hilbert module $L_{0}\left[L_{2}\left(\Omega_{1}\right)\right]$, i. e.,

$$
\left\langle T_{1} f, g\right\rangle_{1}=\left\langle f, T_{1} g\right\rangle_{1}
$$

A system $\left\{f_{\alpha}(x, y)\right\} \subset L_{0}\left[L_{2}\left(\Omega_{1}\right)\right]$ is $\nabla_{1}$-orthogonal system, if $\left\langle f_{\alpha}, f_{\beta}\right\rangle_{1}=\theta, \alpha \neq \beta$. A $\nabla_{1}$-orthogonal system $\left\{f_{\alpha}(x, y)\right\} \subset L_{0}\left[L_{2}\left(\Omega_{1}\right)\right]$ is said to be $\nabla_{1}$-orthonormal system, if $\left\langle f_{\alpha}, f_{\alpha}\right\rangle_{1}=\mathbf{e}$.

Note that, the PIO $T_{1}$ is a good example for cyclically compact operators on KaplanskyHilbert module [7]. 


\section{3. $L_{0}$-Eigenvalue of the Partial Integral Operator $T_{1}$}

In this section we prove the existence of an $L_{0}$-eigenvalue for the PIO. Put $\mathscr{H}=$ $L_{0}\left[L_{2}\left(\Omega_{1}\right)\right]$.

Theorem 3.1. The partial integral operator $T_{1}$ has non zero $L_{0}$-eigenvalue.

$\triangleleft \mathrm{Put}$

$$
\mathscr{D}_{0}=\left\{\omega \in \Omega_{2}: \int_{\Omega_{1}} \int_{\Omega_{1}}\left|k_{1}(x, s, \omega)\right|^{2} d \mu_{1}(x) d \mu_{1}(s)>0\right\} .
$$

Then $\mu_{2}\left(\mathscr{D}_{0}\right)=0$. For each $f \in \mathscr{H}, f \neq \theta$ we define subset $\operatorname{supp}_{\Omega_{2}}(f)$ with positive measure by the following equality

$$
\operatorname{supp}_{\Omega_{2}}(f)=\left\{\omega \in \Omega_{2}:\langle f, f\rangle_{1}(\omega) \neq 0\right\} .
$$

Let $f_{0} \in \mathscr{H},\left\|f_{0}\right\|_{1}(\omega) \neq 0$ for all $\omega \in \mathscr{D}_{0}$ and $T_{1} f_{0} \neq \theta$. It is clear, that $T_{1}^{n} f_{0} \neq \theta$ for all $n \in \mathbb{N}$, as: if

$$
T_{1}^{k} f_{0} \neq \theta, \quad T_{1}^{k+1} f_{0}=\theta, \text { for some } k \geqslant 1
$$

then we get a contradiction

$$
\theta=\left\langle T_{1}^{k+1} f_{0}, T_{1}^{k-1} f_{0}\right\rangle_{1}(\omega)=\left\langle T_{1}^{k} f_{0}, T_{1}^{k} f_{0}\right\rangle_{1}(\omega) \neq \theta .
$$

We construct two sequences $\left\{\widetilde{f}_{k}(x, \omega)\right\}_{k \in \mathbb{N}_{0}},\left\{f_{k}(x, \omega)\right\}_{k \in \mathbb{N}_{0}}$ of functions from the KaplanskyHilbert module $L_{0}\left[L_{2}\left(\Omega_{1}\right)\right]\left(\mathbb{N}_{0}=\mathbb{N} \cup 0\right)$ :

$$
\begin{gathered}
\widetilde{f}_{k}(x, \omega)= \begin{cases}\frac{f_{k}(x, \omega)}{\left\|f_{k}\right\|_{1}(\omega)}, & x \in \Omega_{1}, \omega \in \operatorname{supp}_{\Omega_{2}}\left(f_{k}\right), \\
0, & x \in \Omega_{1}, \omega \in \Omega_{2} \backslash \operatorname{supp}_{\Omega_{2}}\left(f_{k}\right),\end{cases} \\
f_{k+1}(x, \omega)=\left(T_{1} \widetilde{f}_{k}\right)(x, \omega) .
\end{gathered}
$$

It follows from [9] that

$$
\left\|f_{k}\right\|_{1}(\omega) \leqslant\left\|f_{k+1}\right\|_{1}(\omega), \quad k \in \mathbb{N}
$$

and

$$
\left\|f_{k+1}\right\|_{1}(\omega) \cdot\left\|f_{k}\right\|_{1}(\omega)=\left\langle f_{k-1}, f_{k+1}\right\rangle_{1}(\omega)=\left\langle f_{k+1}, f_{k-1}\right\rangle_{1}(\omega), \quad k \in \mathbb{N} .
$$

On the other hand

$$
\left\|T_{1} \widetilde{f}_{k-1}\right\|_{1}(\omega) \leqslant\left\|T_{1}\right\|_{1}(\omega), \quad k \in \mathbb{N},
$$

where $\left\|T_{1}\right\|_{1}(\omega) \in L_{0}\left(\Omega_{2}\right)$ is the $L_{0}\left(\Omega_{2}\right)$ valued norm of the PIO $T_{1}$. Consequently,

$$
\left\|f_{k}\right\|_{1}(\omega) \leqslant\left\|T_{1}\right\|_{1}(\omega), \quad k \in \mathbb{N} .
$$

Thus, for almost all $\omega \in \Omega_{2}$ the sequence $\left\{\left\|f_{k}\right\|_{1}(\omega)\right\}_{k \in \mathbb{N}}$ has a finite limit $\lambda(\omega) \geqslant 0$, i. e.,

$$
\lim _{k \rightarrow \infty}\left\|f_{k}\right\|_{1}(\omega)=\lambda(\omega)
$$


for almost all $\omega \in \Omega_{2}$. We have $\lambda(\omega) \in L_{0}\left(\Omega_{2}\right)$, as $\left\|f_{k}\right\|_{1}(\omega) \in L_{0}\left(\Omega_{2}\right), k \in \mathbb{N}$. From the relation (5) it follows that $\lambda \neq \theta$. Now, we define the family of integral operators $\left\{T_{1}(\omega)\right\}$ on $L_{2}\left(\Omega_{1}\right)$ by

$$
T_{1}(\omega) \varphi(x)=\int_{\Omega_{1}} k_{1}(x, s, \omega) \varphi(s) d \mu_{1}(s), \quad \varphi \in L_{2}\left(\Omega_{1}\right), \omega \in \Omega_{2} .
$$

Then, $T_{1}(\omega)$ is a compact operator on $L_{2}\left(\Omega_{1}\right)$ for almost all $\omega \in \Omega_{2}$. By the compactness of the operator $T_{1}(\omega)$ there exists subsequence $\tilde{f}_{n_{i}}(x, \omega)$ such that $f_{n_{i}+1}(x, \omega)=T_{1}(\omega) \widetilde{f}_{n_{i}}(x, \omega)$ has a limit $g(x, \omega)$ in the $L_{0}$-norm $\|\cdot\|_{1}$. It is clear $g \in \mathscr{H}$ and $g \neq \theta$. Analogously, for each sequence

$$
f_{n_{i}+2}(x, \omega)=T_{1}(\omega) \widetilde{f}_{n_{i}+1}(x, \omega), \quad f_{n_{i}+3}(x, \omega)=T_{1}(\omega) \widetilde{f}_{n_{i}+2}(x, \omega)
$$

we obtain $f_{n_{i}+2} \rightarrow h \in \mathscr{H}$ and $f_{n_{i}+3} \rightarrow \widetilde{h} \in \mathscr{H}$ by the $L_{0}$-norm $\|\cdot\|_{1}$.

Using the relations (6), (7) we obtain

$$
\begin{gathered}
\|\widetilde{h}-g\|_{1}^{2}(\omega)=\lim _{k \rightarrow \infty}\left\|f_{n_{k}+3}-f_{n_{k}+1}\right\|_{1}^{2}(\omega) \\
=\lim _{k \rightarrow \infty}\left\{\left\|f_{n_{k}+3}\right\|_{1}^{2}(\omega)+\left\|f_{n_{k}+1}\right\|_{1}^{2}(\omega)-\left\langle f_{n_{k}+3}, f_{n_{k}+1}\right\rangle_{1}(\omega)-\left\langle f_{n_{k}+1}, f_{n_{k}+3}\right\rangle_{1}(\omega)\right\}=0
\end{gathered}
$$

for almost all $\omega \in \Omega_{2}$ and so $\widetilde{h}=g$. On the other hand, from the equalities

$$
\begin{aligned}
& f_{n_{k}+2}(x, \omega)= \begin{cases}\frac{\left(T_{1}(\omega) f_{n_{k}+1}\right)(x, \omega)}{\left\|f_{n_{k}+1}\right\|_{1}(\omega)}, & x \in \Omega_{1}, \omega \in \operatorname{supp}_{\Omega_{2}}\left(f_{n_{k}+1}\right), \\
0, & x \in \Omega_{1}, \omega \in \Omega_{2} \backslash \operatorname{supp}_{\Omega_{2}}\left(f_{n_{k}+1}\right),\end{cases} \\
& f_{n_{k}+3}(x, \omega)= \begin{cases}\frac{\left(T_{1}(\omega) f_{n_{k}+2}\right)(x, \omega)}{\left\|f_{n_{k}+2}\right\|_{1}(\omega)}, & x \in \Omega_{1}, \omega \in \operatorname{supp}_{\Omega_{2}}\left(f_{n_{k}+2}\right), \\
0, & x \in \Omega_{1}, \omega \in \Omega_{2} \backslash \operatorname{supp}_{\Omega_{2}}\left(f_{n_{k}+2}\right)\end{cases}
\end{aligned}
$$

we have

$$
\begin{aligned}
& \left\|f_{n_{k}+1}\right\|_{1}(\omega) \cdot f_{n_{k}+2}(x, \omega)=\left(T_{1}(\omega) f_{n_{k}+1}\right)(x, \omega), \quad \omega \in \Omega_{2}, \\
& \left\|f_{n_{k}+2}\right\|_{1}(\omega) \cdot f_{n_{k}+3}(x, \omega)=\left(T_{1}(\omega) f_{n_{k}+2}\right)(x, \omega), \quad \omega \in \Omega_{2} .
\end{aligned}
$$

It is clear that

$$
\begin{gathered}
\lim _{k \rightarrow \infty}\left\|f_{n_{k}+1}\right\|_{1}(\omega)=\|g\|_{1}(\omega)=\lim _{k \rightarrow \infty}\left\|f_{n_{k}+2}\right\|_{1}(\omega)=\|h\|_{1}(\omega) \\
=\lim _{k \rightarrow \infty}\left\|f_{n_{k}+3}\right\|_{1}(\omega)=\|\widetilde{h}\|_{1}(\omega)=\lambda(\omega) .
\end{gathered}
$$

From the equalities (8), (9) it follows that

$$
\lambda(\omega) \cdot h(x, \omega)=T_{1}(\omega) g(x, \omega), \quad \lambda(\omega) \cdot \widetilde{h}(x, \omega)=T_{1}(\omega) h(x, \omega),
$$

i. e.,

$$
\left(T_{1} g\right)(x, y)=\lambda(y) \cdot h(x, y), \quad\left(T_{1} h\right)(x, y)=\lambda(y) \cdot g(x, y) .
$$

Hence it follows that

$$
T_{1}(h+g)(x, y)=\lambda(y) \cdot(h+g)(x, y), \quad T_{1}(h-g)(x, y)=-\lambda(y) \cdot(h-g)(x, y) .
$$

We know, that $h \neq \theta, g \neq \theta$. Hence we can conclude that: $h+g \neq \theta$ or $h-g \neq \theta$. It means that the function $\lambda(y)$ is an $L_{0}$-eigenvalue of the PIO $T_{1}$. $\triangleright$ 


\section{Spectral Properties of the Partial Integral Operator $T_{1}$ on the Kaplansky-Hilbert Module $L_{0}\left[L_{2}\left(\Omega_{1}\right)\right]$}

Theorem 4.1. For a PIO $T_{1}$ the following function $\lambda_{0}(\omega)=\sup _{\|g\|_{1}=\mathbf{e}}\left|\left\langle T_{1} g, g\right\rangle_{1}(\omega)\right|$ is nonzero and either $+\lambda_{0}(\omega)$ or $-\lambda_{0}(\omega)$ is $L_{0}$-eigenvalue of the $T_{1}$.

$\triangleleft$ Put

$$
\Omega_{0}=\left\{\omega \in \Omega_{2}: \int_{\Omega_{1}} \int_{\Omega_{1}}\left|k_{1}(x, s, \omega)\right|^{2} d \mu_{1}(x) d \mu_{1}(s)>0\right\} .
$$

From the $T_{1} \neq \theta$ it follows that $\lambda_{0}(\omega) \neq 0$ for all $\omega \in \Omega_{0}$, i. e., $\lambda_{0} \neq \theta$. It is clear, that there is a sequence of $\nabla_{1}$-normal functions $\left\{g_{n}\right\}_{n=1}^{\infty}$, in which a limit exists

$$
(o)-\lim _{n \rightarrow \infty}\left\langle T_{1} g_{n}, g_{n}\right\rangle_{1}(\omega)=\lambda(\omega),
$$

and $\lambda(\omega)$ is a real function on $\Omega_{2}$, where $\lambda(\omega)=+\lambda_{0}(\omega)$ or $-\lambda_{0}(\omega)$. Consequently, $\lambda_{0} \in L_{0}\left(\Omega_{2}\right)$ and $\operatorname{supp}(\lambda)=\Omega_{0}$.

By cyclical compactness of the PIO $T_{1}$ there exists a subsequence $\left\{g_{n_{i}}\right\}_{i=1}^{\infty}$ with

$$
(b o)-\lim _{k \rightarrow \infty}\left(T_{1} g_{n_{k}}\right)(x, y)=h(x, y) \text {. }
$$

Clearly, $\operatorname{supp}_{\Omega_{2}}(h)=\Omega_{0}$. From the equality

$$
\left\|T_{1} g_{n_{k}}-\lambda \cdot g_{n_{k}}\right\|_{1}^{2}=\left\|T_{1} g_{n_{k}}\right\|_{1}^{2}-2 \lambda \cdot\left\langle T_{1} g_{n_{k}}, g_{n_{k}}\right\rangle_{1}+\lambda^{2}
$$

we obtain

$$
\text { (o)- } \lim _{k \rightarrow \infty}\left\|T_{1} g_{n_{k}}-\lambda \cdot g_{n_{k}}\right\|_{1}^{2}=\|h\|_{1}^{2}-\lambda^{2} .
$$

However,

$$
\left\|T_{1} g_{n_{k}}\right\|_{1}(\omega) \leqslant \lambda_{0}(\omega) \cdot\left\|g_{n_{k}}\right\|_{1}(\omega)=|\lambda(\omega)|
$$

Therefore,

$$
\|h\|_{1}(\omega) \leqslant|\lambda(\omega)| .
$$

From this and (11) we have $\|h\|_{1}(\omega)=|\lambda(\omega)|$. Thus,

$$
\text { (o)- } \lim _{k \rightarrow \infty}\left\|T_{1} g_{n_{k}}-\lambda \cdot g_{n_{k}}\right\|_{1}=\theta \text {. }
$$

Hence, it follows that

$$
T_{1} f_{0}=\lambda \cdot f_{0},
$$

where

$$
f_{0}(x, \omega)= \begin{cases}\frac{h(x, \omega)}{\lambda(\omega)}, & x \in \Omega_{1}, \omega \in \operatorname{supp}_{\Omega_{2}}(\lambda), \\ 0, & x \in \Omega_{1}, \omega \in \Omega_{2} \backslash \operatorname{supp}_{\Omega_{2}}(\lambda) . \triangleright\end{cases}
$$

Put

$$
\pi_{0}(\omega)= \begin{cases}1, & \omega \in \operatorname{supp}_{\Omega_{2}}(\lambda) \\ 0, & \omega \in \Omega_{2} \backslash \operatorname{supp}_{\Omega_{2}}(\lambda) .\end{cases}
$$

REMARK 4.1. Every element $\zeta \in L_{0}\left(\Omega_{2}\right), \pi_{0} \zeta=\lambda$ is $L_{0}$-eigenvalue of the PIO $T_{1}$. 
Theorem 4.2. The PIO $T_{1}$ has a finite or countable sequence of $\nabla_{1}$-orthonormal eigenfunctions

$$
\phi_{1}(x, y), \phi_{2}(x, y), \ldots, \phi_{n}(x, y), \ldots
$$

corresponding to a system of real nonzero $L_{0}$-eigenvalues

$$
\lambda_{1}(\omega), \lambda_{2}(\omega), \ldots, \lambda_{n}(\omega), \ldots
$$

where

$$
\left|\lambda_{1}(\omega)\right| \geqslant\left|\lambda_{2}(\omega)\right| \geqslant \ldots \geqslant\left|\lambda_{n}(\omega)\right| \geqslant \ldots
$$

Moreover, for each $f(x, y) \in L_{0}\left[L_{2}\left(\Omega_{1}\right)\right]$ the equality

$$
\|f\|_{1}^{2}(\omega)=(o)-\sum_{k=1}^{\infty}\left|\left\langle f, \phi_{k}\right\rangle_{1}(\omega)\right|^{2}
$$

holds.

$\triangleleft$ Put $\mathscr{H}_{1}=\mathscr{H}$ and $T_{1}^{(1)}=T_{1}$. By the Theorem 4.1 there is such element $\phi_{1}(x, y) \in \mathscr{H}_{1}$ that $T_{1}^{(1)} \phi_{1}=\lambda_{1} \cdot \phi_{1}$, where $\lambda_{1}$ is a real function on $\Omega_{2}$ and $\lambda_{1}(\omega)= \pm \sup _{\|g\|_{1}=\mathbf{e}}\left|\left\langle T_{1} g, g\right\rangle_{1}(\omega)\right|$. We define the Kaplansky-Hilbert submodule $\mathscr{H}_{2}=\mathscr{H}_{1} \ominus_{1}\left\{\phi_{1}\right\}$. It is clear that if $f \in \mathscr{H}_{2}$, then $T_{1}^{(1)} f \in \mathscr{H}_{2}$ from the equality $\left\langle f, \phi_{1}\right\rangle_{1}=\theta$ it follows that

$$
\left\langle T_{1}^{(1)} f, \phi_{1}\right\rangle_{1}=\left\langle f, T_{1} \phi_{1}\right\rangle_{1}=\left\langle f, \lambda_{1} \cdot \phi_{1}\right\rangle_{1}=\theta .
$$

We define an operator $T_{1}^{(2)}$ on the $\mathscr{H}_{2}$ by

$$
T_{1}^{(2)} f=T_{1}^{(1)} f, \quad f \in \mathscr{H}_{2} .
$$

The operator $T_{1}^{(2)}$ is a selfadjoint PIO on the $\mathscr{H}_{2}$. If $T_{1}^{(2)} \neq \theta$, then we apply Theorem 4.1 to the operator $T_{1}^{(2)}$ and find an element $\phi_{2}(x, y) \in \mathscr{H}_{2}$ such that $T_{1}^{(2)} \phi_{2}=\lambda_{2} \cdot \phi_{2}$, where $\lambda_{2}$ is a real function on $\Omega_{2}$ and $\lambda_{2}(\omega)= \pm \sup _{g \in \mathscr{H}_{2},\|g\|_{1}=\mathbf{e}}\left|\left\langle T_{1}^{(2)} g, g\right\rangle_{1}(\omega)\right|$. As $\phi_{2}(x, y) \in \mathscr{H}_{2},\left\|\phi_{2}\right\|_{1}=\mathbf{e}$, we have $\left\langle\phi_{2}, \phi_{1}\right\rangle_{1}=\theta$. Therefore,

$$
\left|\lambda_{2}(\omega)\right|=\sup _{g \in \mathscr{H}_{2},\|g\|_{1}=\mathbf{e}}\left|\left\langle T_{1}^{(1)} g, g\right\rangle_{1}(\omega)\right| \leqslant \sup _{g \in \mathscr{H}_{1},\|g\|_{1}=\mathbf{e}}\left|\left\langle T_{1}^{(1)} g, g\right\rangle_{1}(\omega)\right|=\left|\lambda_{1}(\omega)\right| .
$$

Continuing this process we obtain a sequence of Kaplansky-Hilbert submodules $\mathscr{H}_{k+1}=$ $\mathscr{H}_{k} \ominus_{1}\left\{\phi_{k}\right\}$, where $\phi_{k} \in \mathscr{H}_{k}$ are eigenfunctions of the PIO $T_{1}$ with $T_{1} \phi_{k}=\lambda_{k} \cdot \phi_{k}$.

If $T_{1}^{(n)}$ is a zero operator for some $n \in \mathbb{N}$ then we obtain the finite system $\nabla_{1}$-orthonormal eigenfunctions $\phi_{1}(x, y), \phi_{2}(x, y), \ldots, \phi_{n-1}(x, y)$ corresponding to the system of nonzero $L_{0^{-}}$ eigenvalues $\lambda_{1}(\omega), \lambda_{2}(\omega), \ldots, \lambda_{n-1}(\omega)$, such that

$$
\left|\lambda_{1}(\omega)\right| \geqslant\left|\lambda_{2}(\omega)\right| \geqslant \ldots \geqslant\left|\lambda_{n-1}(\omega)\right|
$$

and

$$
\left|\lambda_{k}(\omega)\right|=\sup _{g \in \mathscr{H}_{k},\|g\|_{1}=\mathbf{e}}\left|\left\langle T_{1}^{(1)} g, g\right\rangle_{1}(\omega)\right| .
$$

If $T_{1}^{(n)} \neq \theta$ for each $n \in \mathbb{N}$ then we obtain an infinite system $\nabla_{1}$-orthonormal eigenfunctions $\left\{\phi_{k}\right\}_{k=1}^{\infty}$ corresponding to the system of $L_{0}$-eigenvalues $\lambda_{k} \neq \theta$. However, the equality

$$
T_{1}(\omega) \phi_{k}(x, \omega)=\lambda_{k}(\omega) \cdot \phi_{k}(x, \omega), \quad k \in \mathbb{N},
$$


is correct for almost all $\omega \in \Omega_{2}$. It follows that $\lim _{k \rightarrow \infty} \lambda_{k}(\omega)=0$ for almost all $\omega \in \Omega_{2}$, because $T_{1}(\omega)$ is a compact operator for almost all $\omega \in \Omega_{2}$.

Let $f=T_{1} h, h \in \mathscr{H}$ and $g=h-\sum_{k=1}^{m}\left\langle h, \phi_{k}\right\rangle_{1} \cdot \phi_{k}$. Here $m$ is the number of eigenfunctions of the system $\left\{\phi_{k}\right\}$ when the system $\left\{\phi_{k}\right\}$ is a finite set, and $m$ is equal to arbitrary natural number otherwise. By the equality

$$
\left\langle g, \phi_{k}\right\rangle_{1}=\theta, \quad k \in\{1,2, \ldots, m\}
$$

we have $g \in \mathscr{H}_{m+1}$. Consequently, we have

$$
\left\|T_{1} g\right\|_{1}^{2}(\omega) \leqslant\left\|T_{1}^{(m+1)}\right\|^{2}(\omega) \cdot\|g\|_{1}^{2}(\omega),
$$

i. e.,

$$
\left\|T_{1} h-\sum_{k=1}^{m}\left\langle h, \phi_{k}\right\rangle_{1} \cdot T_{1} \phi_{k}\right\|_{1}^{2}(\omega) \leqslant\left\|T_{1}^{(m+1)}\right\|^{2}(\omega) \cdot\|g\|_{1}^{2}(\omega) .
$$

We have $\left\langle h, \phi_{k}\right\rangle_{1} \cdot T_{1} \phi_{k}=\left\langle T_{1} h, \phi_{k}\right\rangle_{1} \cdot \phi_{k}$ and $\|g\|_{1} \leqslant\|h\|_{1}$. Hence by the inequality (13) we obtain

$$
\left\|f-\sum_{k=1}^{m}\left\langle f, \phi_{k}\right\rangle_{1} \cdot \phi_{k}\right\|_{1}^{2}(\omega) \leqslant\left\|T_{1}^{(m+1)}\right\|_{1}^{2}(\omega) \cdot\|h\|_{1}^{2}(\omega) .
$$

If the number of elements of the system $\left\{\phi_{k}\right\}$ is equal to $m$ then $T_{1}^{(m+1)}=\theta$ and we have

$$
f=\sum_{k=1}^{m}\left\langle f, \phi_{k}\right\rangle_{1} \cdot \phi_{k}
$$

If the sequence $\left\{\phi_{k}\right\}$ is infinite then from the inequality (14) it follows that

$$
\left\|f-\sum_{k=1}^{m}\left\langle f, \phi_{k}\right\rangle_{1} \cdot \phi_{k}\right\|_{1}^{2}(\omega) \leqslant \lambda_{m+1}^{2}(\omega) \cdot\|h\|_{1}^{2}(\omega),
$$

i. e.,

$$
\theta \leqslant\|f\|_{1}^{2}(\omega)-\sum_{k=1}^{m}\left|\left\langle f, \phi_{k}\right\rangle_{1}(\omega)\right|^{2} \leqslant \lambda_{m+1}^{2}(\omega) \cdot\|h\|_{1}^{2}(\omega) .
$$

Thus as $m \rightarrow \infty$, we get

$$
\|f\|_{1}^{2}(\omega)=(o)-\sum_{k=1}^{\infty}\left|\left\langle f, \phi_{k}\right\rangle_{1}(\omega)\right|^{2} . \triangleright
$$

\section{Decomposition of the Partial Integral Operator $T_{1}$ in Series of $\nabla_{1}$-One-Dimensional Operators}

Definition 5.1. If for an operator $A: \mathscr{H} \rightarrow \mathscr{H}$ there are $\nabla_{1}$-orthonormal functions $\left\{\phi_{k}\right\}_{k=1}^{n} \subset \mathscr{H}$ and some system of functions $\left\{g_{k}\right\}_{k=1}^{n} \subset \mathscr{H}$, such that

$$
A f=\sum_{k=1}^{n}\left\langle f . g_{k}\right\rangle_{1} \cdot \phi_{k}, \quad f \in \mathscr{H}
$$

then the operator $A$ is called the $\nabla_{1}$-n-dimensional operator, here $\mathscr{H}=L_{0}\left[L_{2}\left(\Omega_{1}\right)\right]$. 
Theorem 5.1. For the PIO $T_{1}$ there is a system of $\nabla_{1}$-orthonormal functions $\left\{\phi_{k}(x, y)\right\}$ and a sequence of real $L_{0}\left(\Omega_{2}\right)$-eigenvalues $\lambda_{k}(\omega)$ such that for all $h \in L_{0}\left[L_{2}\left(\Omega_{1}\right)\right]$ the following conditions hold:

$1^{\circ} . h=h_{0}+(b o)-\sum_{k=1}^{\infty}\left\langle h, \phi_{k}\right\rangle_{1} \cdot \phi_{k}, h_{0} \in \operatorname{Ker}\left(T_{1}\right)$.

$2^{\circ} . T_{1} h=(b o)-\sum_{k=1}^{\infty} \lambda_{k} \cdot\left\langle h, \phi_{k}\right\rangle_{1} \cdot \phi_{k}$.

$3^{\circ} .\left|\lambda_{k}(\omega)\right| \geqslant\left|\lambda_{k+1}(\omega)\right|, k \in \mathbb{N}$.

$4^{\circ}$. (o) $-\lim _{k \rightarrow \infty} \lambda_{k}=\theta$.

$\triangleleft$ By Theorem 4.2 there are a system of $\nabla_{1}$-orthonormal functions $\left\{\phi_{k}(x, y)\right\}$ and a sequence of $L_{0}$-eigenvalues $\lambda_{k}(\omega)$ such that $T \phi_{k}=\lambda_{k} \cdot \phi_{k}$ and for each $f=T_{1} h$ we get the equality

$$
f=(b o)-\sum_{k=1}^{\infty}\left\langle f, \phi_{k}\right\rangle_{1} \cdot \phi_{k}
$$

where $\left\langle f, \phi_{k}\right\rangle_{1}=\lambda_{k} \cdot\left\langle h, \phi_{k}\right\rangle_{1}$.

Thus, for all $h \in \mathscr{H}$

$$
T_{1} h=(b o)-\sum_{k=1}^{\infty} \lambda_{k} \cdot\left\langle h, \phi_{k}\right\rangle_{1} \cdot \phi_{k} .
$$

If we denote $h_{0}=h-(b o)-\sum_{k=1}^{\infty}\left\langle h, \phi_{k}\right\rangle_{1} \cdot \phi_{k}$, then

$$
h=h_{0}+(b o)-\sum_{k=1}^{\infty}\left\langle h, \phi_{k}\right\rangle_{1} \cdot \phi_{k}, \quad T_{1} h_{0}=\theta .
$$

The properties $3^{\circ}$ and $4^{\circ}$ follows from the Theorem 4.2 . Theorem 5.1 can also be proven by using Theorem 3.5 in the article of A. G. Kusraev [10]. $\triangleright$

Theorem 5.2. For all positive functions $\varepsilon(\omega) \in L_{0}\left(\Omega_{2}\right), \mu_{2}\left(\Omega_{2} \backslash \operatorname{supp}(\varepsilon)\right)=0$ there exist a $\nabla_{1}$-finite dimensional operator $\mathscr{T}_{1}^{\varepsilon}$ on the Kaplansky-Hilbert module $L_{0}\left[L_{2}\left(\Omega_{1}\right)\right]$, such that $\left\|T_{1}-\mathscr{T}_{1}^{\varepsilon}\right\|_{1}(\omega)<\varepsilon(\omega)$.

$\triangleleft$ By the Theorem 5.1 there is a system of $\nabla_{1}$-orthonormal functions $\left\{\phi_{k}(x, y)\right\}$ and a sequence of $L_{0}$-eigenvalues $\lambda_{k}(\omega)$ for which the properties $1^{\circ}-4^{\circ}$ hold. We define the $\nabla_{1^{-}}$ finite dimensional operator $\mathscr{T}_{1}^{\varepsilon}$ :

$$
\mathscr{T}_{1}^{\varepsilon} h=\sum_{k=1}^{n} \lambda_{k} \cdot\left\langle h, \phi_{k}\right\rangle_{1} \cdot \phi_{k}
$$

It follows that

$$
\left\|T_{1} h-\mathscr{T}_{1}^{\varepsilon} h\right\|_{1}^{2}(\omega) \leqslant \lambda_{n+1}^{2}(\omega) \cdot\left\{\left|\left\langle h, \phi_{k+1}\right\rangle_{1}(\omega)\right|^{2}+\left|\left\langle h, \phi_{k+2}\right\rangle_{1}(\omega)\right|^{2}+\ldots\right\} \leqslant \lambda_{k}^{2}(\omega)\|h\|_{1}^{2}(\omega) .
$$

Hence, for $\left|\lambda_{n+1}(\omega)\right|<\varepsilon(\omega)$ we have $\left\|T_{1}-\mathscr{T}_{1}^{\varepsilon}\right\|_{1}(\omega)<\varepsilon(\omega)$. $\triangleright$

\section{Decomposition of the Partial Integral Operator $T_{2}$ in Series of $\nabla_{2}$-One-Dimensional Operators}

We denote by $L_{0}\left[L_{2}\left(\Omega_{2}\right)\right]$ the set of equivalence classes of all complex measurable functions $f(x, y)$ on $\Omega_{1} \times \Omega_{2}$, which satisfied the condition: the integral

$$
\psi(x)=\int_{\Omega_{2}}|f(x, y)|^{2} d \mu_{2}(y)
$$

exist for almost all $x \in \Omega_{1}$ and $\psi \in L_{0}\left(\Omega_{1}\right)$. 
We define $L_{0}\left(\Omega_{1}\right)$-valued inner product on $L_{0}\left[L_{2}\left(\Omega_{2}\right)\right]$ by

$$
\langle f, g\rangle_{2}=\int_{\Omega_{1}} f(x, t) \overline{g(x, t)} d \mu_{2}(t) .
$$

For each $f \in L_{0}\left[L_{2}\left(\Omega_{2}\right)\right]$ we define $L_{0}$-norm: $\|f\|_{2}(v)=\sqrt{\langle f, f\rangle_{2}(v)}$. Then $L_{0}\left[L_{2}\left(\Omega_{2}\right)\right]$ is a Banach-Kantorovich space over $L_{0}\left(\Omega_{1}\right)$. Consequently, the space $L_{0}\left[L_{2}\left(\Omega_{2}\right)\right]$ is a KaplanskyHilbert module over $L_{0}\left(\Omega_{1}\right)$ with the inner product $\langle\cdot, \cdot\rangle_{2}(v)$.

Let $T_{2}$ be an operator in the Kaplansky-Hilbert module $L_{0}\left[L_{2}\left(\Omega_{2}\right)\right]$ over $L_{0}\left(\Omega_{1}\right)$ given by the formula

$$
\left(T_{2} f\right)(x, y)=\int_{\Omega_{2}} k_{2}(x, t, y) f(x, t) d \mu_{2}(t) .
$$

Here, $k_{2}(x, t, y)$ is measurable function on $\Omega_{1} \times \Omega_{2}^{2}$.

Assume that the kernel $k_{2}(x, s, y)$ of the integral operator $T_{2}$ satisfies the condition

$$
\int_{\Omega_{2}} \int_{\Omega_{2}}\left|k_{2}(x, t, y)\right|^{2} d \mu_{2}(t) d \mu_{2}(y) \in L_{0}\left(\Omega_{1}\right) .
$$

Then, the operator $T_{2}$ is linear and $L_{0}\left(\Omega_{1}\right)$-bounded operator on $L_{0}\left[L_{2}\left(\Omega_{2}\right)\right]$. If the kernel $k_{2}(x, s, y)$ satisfy of the condition $k_{2}(x, t, y)=\overline{k_{2}(x, y, t)}$, then the operator $T_{2}$ is a self-adjoint operator on the Kaplansky-Hilbert module $L_{0}\left[L_{2}\left(\Omega_{2}\right)\right]$, i. e.,

$$
\left\langle T_{2} f, g\right\rangle_{2}=\left\langle f, T_{2} g\right\rangle_{2} .
$$

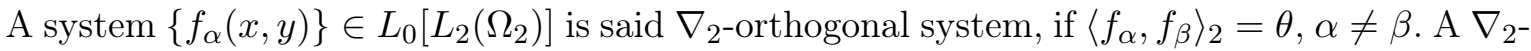
orthogonal system $\left\{f_{\alpha}(x, y)\right\} \subset L_{0}\left[L_{2}\left(\Omega_{2}\right)\right]$ is said $\nabla_{2}$-orthonormal system, if $\left\langle f_{\alpha}, f_{\alpha}\right\rangle_{2}=\mathbf{e}$.

Note that, the PIO $T_{2}$ is cyclically compact on the Kaplansky-Hilbert module $L_{0}\left[L_{2}\left(\Omega_{2}\right)\right][7]$.

Theorem 6.1. For the PIO $T_{2}$ there is a system of $\nabla_{2}$-orthonormal functions $\left\{\psi_{k}(x, y)\right\}$ and a sequence of real $L_{0}\left(\Omega_{1}\right)$-eigenvalues $\zeta_{k}(v)$ such that, for all $h \in L_{0}\left[L_{2}\left(\Omega_{2}\right)\right]$ the following hold:

$1^{\circ} \cdot h=h_{0}+(b o)-\sum_{k=1}^{\infty}\left\langle h, \psi_{k}\right\rangle_{1} \cdot \psi_{k}, h_{0} \in \operatorname{Ker}\left(T_{2}\right) ;$

$2^{\circ} \cdot T_{2} h=(b o)-\sum_{k=1}^{\infty} \zeta_{k} \cdot\left\langle h, \psi_{k}\right\rangle_{1} \cdot \psi_{k}$, where

$3^{\circ} .\left|\zeta_{k}(v)\right| \geqslant\left|\zeta_{k+1}(v)\right|, k \in \mathbb{N}$;

$4^{\circ} .(o)-\lim _{k \rightarrow \infty} \zeta_{k}=\theta$.

\section{References}

1. Appell, J., Kalitvin, A. S. and Zabrejko, P. P. Partial Integral Operators and Integro-Differential Equations, New York, Basel, 2000, 578 p.

2. Eshkabilov, $\mathrm{Yu}$. Kh. On a Discrete "Three-Particle" Schrodinger Operator in the Hubbard Model, Theor. Math. Phys., 2006, vol. 149, no. 2, pp. 1497-1511. DOI: 10.1007/s11232-006-0133-2.

3. Eshkabilov, Yu. Kh. and Kucharov, R. R. Essential and Discrete Spectra of the Three-Particle Schrödinger Operator on a Lattice, Theor. Math. Phys., 2012, vol. 170, no. 3, pp. 341-353. DOI: 10.1007/s11232012-0034-5.

4. Eshkabilov, Yu. Kh. The Efimov Effect for a Model "Three-Particle" Discrete Schrödinger Operator, Theor. Math. Phys., 2010, vol. 164, no. 1, pp. 896-904. DOI: 10.1007/s11232-010-0071-x.

5. Eshkabilov, Yu. Kh. Spectra of Partial Integral Operators with a Kernel of Three Variables, Central European J. Math., 2008, vol. 6, no. 1, pp. 149-157. DOI: 10.2478/s11533-008-0010-3.

6. Kusraev, A. G. Dominated Operators, Dordrecht etc., Kluwer Academic Publishers, 2000, 445p. 
7. Kudaybergenov, K. K. $\nabla$-Fredholm Operators in Banach-Kantorovich Spaces, Methods Func. Anal. Topology, 2006, vol. 12, no. 3, pp. 234-242.

8. Sarymsakov, T. A. Polupolya $i$ teoriya veroyatnostej [Semifields and Probability Theory], Tashkent, Fan, 1980 (in Russian).

9. Akhiezer, N. I. and Glazman, I. M. Teoriya linejnyh operatorov v gilbertovom prostranstve [Theory of Linear Operators in Hilbert Space], Moskva, Nauka, 1966, 544 p. (in Russian).

10. Kusraev, A. G. Cyclically Compact Operators in Banach Spaces. Vladikavkaz Math. J., 2000, vol. 2, no. 1 , pp. 10-23.

Received January 18, 2021

Yusup KH. EshKabILOV

Karshi State University,

17 Kuchabag St., Karshi 180119, Uzbekistan,

Professor

E-mail: yusup62@mail.ru

RAMZIDDIN R. KUCHAROV

National University of Uzbekistan,

4 University St., Tashkent 100174, Uzbekistan,

Associate Professor

E-mail: ramz3364647@yahoo.com

Владикавказский математический журнал 2021, Том 23, Выпуск 3, С. 80-90

\title{
ЧАСТИЧНО ИНТЕГРАЛЬНЫЕ ОПЕРАТОРЫ ТИПА ФРЕДГОЛЬМА В МОДУЛЕ КАПЛАНСКОГО - ГИЛЬБЕРТА НАД $L_{0}$
}

\author{
Эшкабилов Ю. Х. ${ }^{1}$, Кучаров Ю. Х. ${ }^{2}$ \\ ${ }^{1}$ Каршинский государственный университет, Узбекистан, 180119, Карши, ул. Кучабаг, 17; \\ ${ }^{2}$ Национальный университет Узбекистана, Узбекистан, 100174, Ташкент, ул. Университетская, 4 \\ E-mail: yusup62@mail.ru, ramz3364647@yahoo.com
}

Посвящается 80-летию профессора
Стефана Григорвевича Самко

Аннотация. В статье изучаются некоторые характеристические свойства самосопряженных частично интегральных операторов типа Фредгольма в модуле Капланского - Гильберта $L_{0}\left[L_{2}\left(\Omega_{1}\right)\right]$ над $L_{0}\left(\Omega_{2}\right)$. Используется математический инструментарий из теории модулей Капланского - Гильберта. В модуле Капланского - Гильберта $L_{0}\left[L_{2}\left(\Omega_{1}\right)\right]$ над $L_{0}\left(\Omega_{2}\right)$ рассматриваются частично интегральные операторы типа Фредгольма $T_{1}\left(\Omega_{1}\right.$ и $\Omega_{2}$ - замкнутые ограниченные множества в $\mathbb{R}^{\nu_{1}}$ и $\mathbb{R}^{\nu_{2}}, \nu_{1}, \nu_{2} \in \mathbb{N}$ соответственно). В работе доказано существование $L_{0}\left(\Omega_{2}\right)$-собственных значений, отличных от нуля для любого самосопряженного частично интегрального оператора типа Фредгольма $T_{1}$; более того, показано существование конечного или счетного числа вещественных $L_{0}\left(\Omega_{2}\right)$-собственных значений. В последнем случае, последовательности $L_{0}\left(\Omega_{2}\right)$-собственных значений порядково сходятся к нулевой функции. Установлена также теорема о разложимости оператора $T_{1}$ в ряд по $\nabla_{1}$ одномерным операторам.

Ключевые слова: частично интегральный оператор, модуль Капланского - Гильберта, $L_{0}$-собственное значение.

Mathematical Subject Classification (2010): 45A05, 47A10, 47G10, 45P05, 45B05, $45 \mathrm{C} 05$.

Образец цитирования: Eshkabilov, Yu. Kh. and Kucharov, R. R. Partial Integral Operators of Fredholm Type on Kaplansky-Hilbert Module over $L_{0} / /$ Владикавк. мат. журн.-2021.- T. 23, № 3.C. 80-90 (in English). DOI: 10.46698/w5172-0182-0041-c. 